\title{
Singular Measures Without Restrictive Intervals *
}

\author{
Stewart D. Johnson \\ Department of Mathematics, Cornell University, Ithaca, NY 14850, USA
}

\begin{abstract}
For the transformation $T:[0,1] \mapsto[0,1]$ defined by $T(x)=\lambda x(1-x)$ with $0 \leqq \lambda \leqq 4$, a $\lambda$ is shown to exist for which $T$ has no restrictive intervals, hence is sensitive to initial conditions, but for which no finite absolutely continuous invariant measure exists for $T$.
\end{abstract}

\section{Introduction}

A restrictive interval $R$ for a one dimensional transformation $T$ is one which eventually maps into itself; $T^{n}(R) \subset R$ for some $n$, the least such $n$ being called the period of the interval. In the case where $T$ is unimodal with negative Schwarzian derivative, John Guckenheimer showed that the absence of a restrictive interval implied an expansion property called sensitivity to initial conditions: $\exists \varepsilon \forall$ intervals $I \exists N$ such that $l\left(T^{N}(I)\right)>\varepsilon$. The same conclusion can be drawn in the case of a finite number of nested restrictive intervals by inducing on the smallest such interval. Thus a dichotomy exists for such maps $T$ not having a period orbit; either $T$ has sensitivity to initial conditions or there exists an infinite nested sequence of restrictive intervals creating an attracting invariant Cantor set upon which $T$ is 1 -1. See Guckenheimer [5], or Collet and Eckmann [2], for a detailed exposition of these ideas.

Of interest is how the structure of invariant measures correspond to this dichotomy. Any invariant Cantor set will support an invariant measure, although it is not clear whether the Cantor sets in this context can have positive lebesgue measure. The question has been posed (Jakobson [6], Guckenheimer [5]) as to whether the absence of a restrictive interval is sufficient to conclude the existence of an absolutely continuous invariant measure. This is shown not to be the case in the following example.

* Supported by the U.S. Army Research Office through the Mathematical Sciences Institute of Cornell University 


\section{Terms and Concepts}

The map to be considered is the quadratic map $T_{\lambda}(x)=\lambda x(1-x)$ which for $0 \leqq \lambda \leqq 4$ maps $[0,1]$ into itself.

Any periodic point $r_{\lambda_{0}}$ of $T_{\lambda_{0}}$ with period $n_{0}$ is a fixed point for $T_{\lambda_{0}}^{n_{0}}$, and if the graph of $T_{\lambda_{0}}^{n_{0}}$ is not tangent to the line $y=x$ at $r_{\lambda_{0}}$ then the point of intersection of the graph and the line will move continuously with small perturbations in $\lambda$. In this case there will exist a closed parameter interval $\left[\lambda_{a}, \lambda_{c}\right]$ containing $\lambda_{0}$ and a periodic point $r_{\lambda}$ which varies continuously for $\lambda \in\left[\lambda_{a}, \lambda_{c}\right]$ such that $r_{\lambda}=r_{\lambda_{0}}$ when $\lambda=\lambda_{0}$.

Let $R_{\lambda_{0}}$ be a restrictive interval of period $n_{0}$ for $T_{\lambda_{0}}$ and assume that $T_{\lambda_{0}}$ has no attracting periodic orbit. Define the interval orbit of $R_{\lambda_{0}}$ to be $\operatorname{Iorb}\left(R_{\lambda_{0}}\right)$ $=\bigcup_{k=0}^{n_{0}-1} T_{\lambda_{0}}^{k}\left(R_{\lambda_{0}}\right)$. One of $R_{\lambda_{0}}, T_{\lambda_{0}}\left(R_{\lambda_{0}}\right), \ldots, T_{\lambda_{0}}^{n_{0}-1}\left(R_{\lambda_{0}}\right)$ must contain the critical point $x=\frac{1}{2}\left[4\right.$, Theorem 2.6] so it may be assumed that $\frac{1}{2} \in R_{\lambda_{0}}$. It will be assumed that $R_{\lambda_{0}}$ is maximal such that $T_{\lambda}^{n_{0}}\left(R_{\lambda_{0}}\right) \subset R_{\lambda_{0}}$, hence one endpoint of $R_{\lambda_{0}}$ will be a periodic point $r_{\lambda_{0}}$ of period $n_{0}$ and the other endpoint will be the symmetric point $r_{\lambda_{0}}^{\prime}=1-r_{\lambda_{0}}$. It is possible to choose $R_{\lambda_{0}}$ so that there is a non-trivial parameter interval in which the points $r_{\lambda}$ and $r_{\lambda}^{\prime}$ vary continuously, creating a continuously varying restrictive interval $R_{\lambda}=\left[r_{\lambda}, r_{\lambda}^{\prime}\right]$ (see [5]). The largest parameter interval $\left[\lambda_{a}, \lambda_{b}\right]$ for which $R_{\lambda}$ is restrictive will be called the "window" of $R_{\lambda}$ (following the terminology of May [9]). For $\lambda$ at the right-hand endpoint of the window, either $r_{\lambda}$ is attracting from one side or $T_{\lambda}^{n_{0}}$ maps $R_{\lambda} 2-1$ and onto itself. In the latter case $R_{\lambda}$ and $\lambda$ will be called "critical" (see Fig. 1). If $\lambda_{b}$ is critical then there will exist $\lambda>\lambda_{b}$ arbitrarily close to $\lambda_{b}$ for which $R_{\lambda}$ is no longer restrictive and $T_{\lambda}^{n_{0}}\left(R_{\lambda}\right)$ will contain a neighborhood of the point $r_{\lambda}^{\prime}$ (see Fig. 2). For such a $\lambda$ the interval orbit will still be taken to be $\operatorname{Iorb}\left(R_{\lambda}\right)=\bigcup_{k=0}^{n_{0}-1} T_{\lambda}^{k}\left(R_{\lambda}\right)$.

Theorem. For $T_{\lambda}=\lambda x(1-x)$ mapping $[0,1]$ into itself, there exists a parameter value $\lambda_{\infty}$ such that $T_{\lambda_{\infty}}$ has no restrictive intervals and any finite invariant measure for $T_{\lambda_{\infty}}$ is singular.

Fig. 1

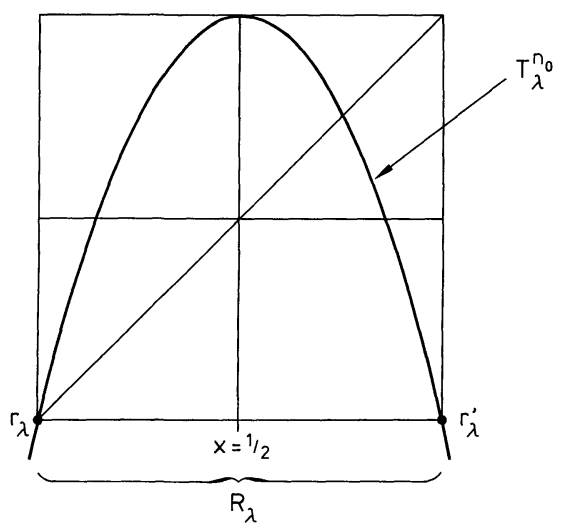


Fig. 2

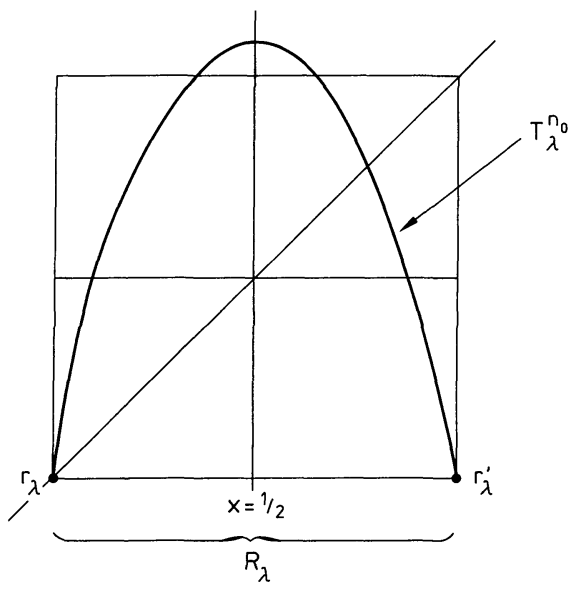

\section{Outline of Proof}

The orbit of a restrictive interval $R_{\lambda}$ attracts almost every point [5, Proposition 2.8, Theorem 3.1] hence any continuous measure for $T_{\lambda}$ will have support contained in $\operatorname{I} \operatorname{orb}\left(R_{\lambda}\right)$. If $\lambda_{b}$ is a critical endpoint of the window $\left[\lambda_{a}, \lambda_{b}\right]$ for $R_{\lambda}$ then for $\lambda$ near $\lambda_{b}$, the expected time it takes a point to land in $R_{\lambda}$ does not change much with respect to small perturbations in $\lambda$. For $\lambda>\lambda_{b}$ the expected time for a point to leave $\operatorname{I} \operatorname{orb}\left(R_{\lambda}\right)$ can be made arbitrarily large by taking $\lambda$ sufficiently close to $\lambda_{b}$. Thus continuous measures can be made to have a preponderance of mass in I orb $\left(R_{\lambda}\right)$ by restricting $\lambda$ close to $\lambda_{b}$. Lemma 1 will show that for $\lambda>\lambda_{b}$ and sufficiently close to $\lambda_{b}$ there will exist a set $P_{\lambda}$ with $l\left(P_{\lambda}^{c}\right)$ arbitrarily small such that $T_{\lambda}^{N}\left(P_{\lambda}\right) \subset \operatorname{Iorb}\left(R_{\lambda}\right)$ for some $N$. Then any invariant measure for $T_{\lambda}$ will have at least half of its mass in $P_{\lambda}^{c} \cup \operatorname{Iorb}\left(R_{\lambda}\right)$.

Lemma 2 is a proof that for any $\varepsilon>0,\left(\lambda_{b}, \lambda_{b}+\varepsilon\right)$ will contain a value $\lambda$ for which $T_{\lambda}$ has a critical restrictive interval $Q_{\lambda}$ with $l\left(\operatorname{Iorb}\left(Q_{\lambda}\right)\right)$ arbitrarily small. Such a parameter value is constructed by consideration of the induced map of $T_{\lambda}^{n_{0}}$ on $R_{\lambda}$ for $\lambda$ slightly larger than $\lambda_{b}$. This induced map will consist of symmetric monotone branches (possibly infinitely many) and a central folded piece (see Fig. 3 and refer

Fig. 3

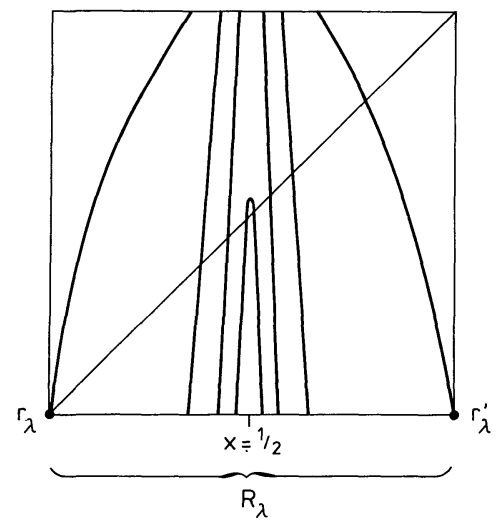


to Guckenheimer [4]). As the central folded piece moves across the line $y=x$ a critical restrictive interval is created. The measure of the orbit of the new restrictive interval is controlled by controlling the size of the new restrictive interval and considering how long its orbit stays close to the orbit of $r_{\lambda}$.

The proof of the theorem consists of using these two lemmas to demonstrate the existence of a sequence of critical parameter values $\lambda_{i}$ with corresponding neighborhoods $\eta_{i}$ such that for $\lambda \in \eta_{i}$ any continuous invariant measure for $T_{\lambda}$ will have a majority of its mass in the corresponding interval orbit (or in a very small set). The neighborhoods $\eta_{i}$ will be constructed to nest down to a point $\lambda_{\infty}$ in the complement of all windows for which any invariant measure for $T_{\lambda_{\infty}}$ is necessarily singular.

The reader is urged to compare this example to Arnold's example for homeomorphisms of the circle (Arnold [1], Cornfeld et al. [3]).

\section{Construction of the Example}

Lemma 1. Suppose $\lambda_{b}$ is a critical endpoint of a window $\left[\lambda_{a}, \lambda_{b}\right]$ for a restrictive interval $R_{\lambda}$ of order $n_{0} . \forall \sigma>0 \exists \delta \forall \lambda \in\left(\lambda_{b}, \lambda_{b}+\delta\right) \exists P_{\lambda}, \exists M$ such that $l\left(P_{\lambda}^{c}\right)<\sigma$ and $T_{\lambda}^{M}\left(P_{\lambda}\right) \subset \operatorname{Iorb}\left(R_{\lambda}\right)$.

Proof. Let any $\sigma>0$ be given and take $\delta^{\prime}$ sufficiently small that $R_{\lambda}=\left[r_{\lambda}, r_{\lambda}^{\prime}\right]$ is defined for $\lambda \in\left[\lambda_{a}, \lambda_{b}+\delta^{\prime}\right)$. Since $\frac{1}{2} \in R_{\lambda}$, almost every point $x$ eventually lands in $R_{\lambda}$ [5, Proposition 2.8, Theorem 3.1] hence $\exists N$ such that $l\left(\bigcup_{k=0}^{N} T_{\lambda}^{-k}\left(R_{\lambda}\right)\right)>1-\frac{\sigma}{2}$ for $\lambda \in\left(\lambda_{b}, \lambda_{b}+\delta^{\prime}\right)$. By continuity, the set $\left\{x \in R_{\lambda}: T_{\lambda}^{n_{0}}(x) \notin R_{\lambda}\right\}$ can be made arbitrarily small by restricting $\lambda>\lambda_{b}$ sufficiently close to $\lambda_{b}$. Hence so can the sets $\left\{x \in R_{\lambda}\right.$ : $\left.\bigcup_{k=0}^{N} T_{\lambda}^{k}(x) \not \subset \operatorname{Iorb}\left(R_{\lambda}\right)\right\}$ and $\bigcup_{i=0}^{N} T_{\lambda}^{i}\left\{x \in R_{\lambda}: \bigcup_{k=0}^{N} T_{\lambda}^{k}(x) \not \subset \operatorname{Iorb}\left(R_{\lambda}\right)\right\}$. Thus the set $E_{\lambda}=\left\{x \in \bigcup_{k=0}^{N} T_{\lambda}^{-k}\left(R_{\lambda}\right): T_{\lambda}^{N}(x) \notin \mathrm{I}\right.$ orb $\left.\left(R_{\lambda}\right)\right\}$ can be made smaller than $\frac{\sigma}{2}$ by restricting $\lambda \in\left(\lambda_{b}, \lambda_{b}+\delta\right)$ for some $\delta<\delta^{\prime}$ sufficiently small. The set $P_{\lambda}=\bigcup_{k=0}^{N} T_{\lambda}^{-k}\left(R_{\lambda}\right)-E_{\lambda}$ has a complement of lebesgue measure less than $\sigma$ and $T_{\lambda}^{N}\left(P_{\lambda}\right) \subset \operatorname{I} \operatorname{orb}\left(R_{\lambda}\right)$.

Thus $P_{\lambda}-\operatorname{Iorb}\left(R_{\lambda}\right)$ is mapped into $P_{\lambda}^{c} \cup \operatorname{I}$ orb $\left(R_{\lambda}\right)$ under $T_{\lambda}^{M}$ for $\lambda \in\left(\lambda_{b}, \lambda_{b}+\delta\right)$. If $\mu$ is any invariant unit measure for $T_{\lambda}$ and $S$ is a set such that $T_{\lambda}^{M}(S) \subset S^{c}$, then $\mu(S)$ $\leqq \mu\left(T_{\lambda}^{-M}\left(S^{c}\right)\right)=\mu\left(S^{c}\right)$ and therefore $\mu\left(S^{c}\right) \geqq \frac{1}{2}$. Thus for $\lambda \in\left(\lambda_{b}, \lambda_{b}+\delta\right)$, any invariant measure for $T_{\lambda}$ has at least half of its mass in $P_{\lambda}^{c} \cup \operatorname{Iorb}\left(R_{\lambda}\right)$.

Lemma 2. Suppose $\lambda_{b}$ is a critical endpoint of a window $\left[\lambda_{a}, \lambda_{b}\right]$ for a restrictive interval $R_{\lambda}$ of order $n_{0} . \forall \varepsilon>0, \forall \sigma>0 \exists$ a critical restrictive interval $Q_{\lambda^{\prime}}$ with parameter value $\lambda^{\prime}$ such that $\lambda^{\prime} \in\left(\lambda_{b}, \lambda_{b}+\varepsilon\right)$ and $l\left(\operatorname{Iorb}\left(Q_{\lambda^{\prime}}\right)\right)<\sigma$.

Proof. For every repelling periodic point $r_{\lambda}$ of period $n_{0}$ there will exist a sequence $b_{i} \rightarrow r_{\lambda}$ of preimages of $\frac{1}{2}$ such that $T_{\lambda}^{n_{0}}\left(b_{i}\right)=b_{i-1}$, and containing each such $b_{i}$ will be a preimage $F_{i}$ of $R_{\lambda_{a}}$. If $\frac{1}{2}$ is not periodic then these preimages will vary smoothly under sufficiently small perturbations in $\lambda$. Thus for $\lambda$ restricted to a sufficiently 
small neighborhood of $\lambda_{b}$ there will exist a sequence $F_{\lambda, i}$ of preimages of $R_{\lambda}$ which vary continuously in $\lambda$ and are such that $T_{\lambda}\left(F_{\lambda, i}\right)=F_{\lambda, i-1}$ with $\operatorname{dist}\left(F_{\lambda, i}, r_{\lambda}\right) \rightarrow 0$ as $i \rightarrow \infty$.

Since the window $\left[\lambda_{a}, \lambda_{b}\right]$ is taken to be maximal, $\exists \lambda_{c}>\lambda_{b}$ and arbitrarily close to $\lambda_{b}$ such that $T_{\lambda_{c}}^{n_{0}}\left(R_{\lambda_{c}}\right)$ contains a neighborhood of $r_{\lambda}^{\prime}$, making $T_{\lambda_{c}}^{2 n_{0}}\left(R_{\lambda_{c}}\right)$ $\supset\left(r_{\lambda_{c}}-\delta, r_{\lambda_{c}}\right)$ for some $\delta>0$. Let such a $\lambda_{c}$ be chosen sufficiently close to $\lambda_{b}$ that the sequence $F_{\lambda, i}$ is continuously varying for $\lambda \in\left(\lambda_{b}, \lambda_{c}\right)$. It may be assumed that $\delta<\sigma$, and since $\operatorname{dist}\left(F_{\lambda, i}, r_{\lambda}\right) \rightarrow 0$ the sequence may be reindexed so that $F_{\lambda, 0} \in\left(r_{\lambda}-\delta, r_{\lambda}\right)$ for $\lambda \in\left(\lambda_{b}, \lambda_{c}\right)$. Let $m_{0}$ be such that $T_{\lambda_{a}}^{m_{0}}\left(F_{\lambda_{a}, 0}\right)=R_{\lambda_{a}}$. Thus a chain $T_{\lambda_{a}}^{m_{0}-1}\left(F_{\lambda_{a}, 0}\right)$, $T_{\lambda_{a}}^{m_{0}-2}\left(F_{\lambda_{a}, 0}\right), \ldots, T_{\lambda_{a}}\left(F_{\lambda_{a}, 0}\right), F_{\lambda_{a}, 0}, F_{\lambda_{a}, 1}, \ldots$ of preimages of $R_{\lambda_{a}}$ is defined. The infinite part $F_{\lambda_{a}, 0}, F_{\lambda_{a}, 1}, F_{\lambda_{a}, 2}, \ldots$ of this chain is contained in $T_{\lambda_{c}}^{2 n_{0}}\left(R_{\lambda_{c}}\right)$ and has lebesgue measure less than $\sigma$.

Define $p_{\lambda, i}$ and $q_{\lambda, i}$ such that $F_{\lambda, i}=\left[p_{\lambda, i}, q_{\lambda, i}\right]$. For each $i$, there exists a parameter interval $\left[\lambda_{i}^{\prime}, \lambda_{i}^{\prime \prime}\right] \subset\left(\lambda_{b}, \lambda_{c}\right)$ such that $T_{\lambda}^{2 n_{0}}\left(\frac{1}{2}\right) \in F_{\lambda, i}$ for $\lambda \in\left[\lambda_{i}^{\prime}, \lambda_{i}^{\prime \prime}\right]$ with

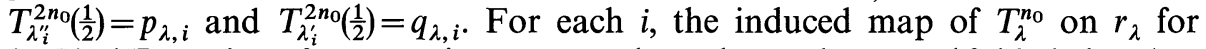
$\lambda \in\left[\lambda_{i}^{\prime}, \lambda_{i}^{\prime \prime}\right]$ consists of symmetric monotone branches and a central folded piece (see Fig. 3). The tip of the central folded piece moves across the interval $R_{\lambda}$ as $\lambda$ moves across the interval $\left[\lambda_{i}^{\prime}, \lambda_{i}^{\prime \prime}\right]$. As the tip moves across the line $y=x$ there will exist a critical parameter value $\lambda_{i} \in\left[\lambda_{i}^{\prime}, \lambda_{i}^{\prime \prime}\right]$ with corresponding critical restrictive interval $Q_{\lambda_{i}}$.

It is to be shown that $l\left(\operatorname{Iorb}\left(Q_{i}\right)\right)$ can be made arbitrarily small. With $m_{0}$ such that $T_{\lambda_{a}}^{m_{0}}\left(F_{\lambda, 0}\right)=R_{\lambda}$, then $Q_{i}$ has period $n_{0}(2+i)+m_{0}$ and the orbit of such a $Q_{i}$ is contained in the chain $T_{\lambda_{a}}^{m_{0}-1}\left(F_{\lambda_{a}, 0}\right), \quad T_{\lambda_{a}}^{m_{0}-2}\left(F_{\lambda_{a}, 0}\right), \ldots, T_{\lambda_{a}}\left(F_{\lambda_{a}, 0}\right)$, $F_{\lambda_{a}, 0}, F_{\lambda_{a}, 1}, F_{\lambda_{a}, 2}, \ldots$. The idea is that by making $Q_{i}$ sufficiently small, its orbit will form a small fraction of the finite part of the chain $T_{\lambda_{a}}^{m_{0}-1}\left(F_{\lambda_{a}, 0}\right)$, $T_{\lambda_{a}}^{m_{0}-2}\left(F_{\lambda_{a}, 0}\right), \ldots, T_{\lambda_{a}}\left(F_{\lambda_{a}, 0}\right)$, and the infinite part of the chain is already bounded by $\sigma$. Specifically, since $Q_{i} \subset\left\{x \in R_{\lambda_{i}}: T_{\lambda_{i}}^{n_{0}}(x) \notin R_{\lambda_{i}}\right\}$ which becomes small as $\lambda_{i} \rightarrow \lambda_{b}$, then $l\left(Q_{i}\right) \rightarrow 0$ and $l\left(T_{\lambda_{i}}^{n_{0}}\left(Q_{i}\right)\right) \rightarrow 0$ as $i \rightarrow \infty$. The set $\bigcup_{k=2}^{i+2} T_{\lambda_{i}}^{n_{0} k}\left(Q_{i}\right) \subset\left(r_{\lambda_{i}}-\delta, r_{\lambda_{i}}\right)$, hence $l\left(\bigcup_{k=2}^{i+2} T_{\lambda_{i}}^{n_{0} k}\left(Q_{i}\right)\right)<\delta<\sigma$ and $\sigma$ may be chosen arbitrarily small. Since $T_{\lambda_{i}}^{\prime}(x)$ is bounded from 0 for $x \notin R_{\lambda_{i}}, l\left(\bigcup_{k=0}^{n_{0}(2+i)} T_{\lambda_{i}}\left(R_{\lambda_{i}}\right)\right)$ can be made arbitrarily small by making $l\left(\bigcup_{k=0}^{2+i} T_{\lambda_{i}}^{n_{0}}\left(R_{\lambda_{i}}\right)\right)$ sufficiently small. Finally, $\bigcup_{k=n_{0}(2+i)+1}^{n_{0}(2+i)+m_{0}-1} T_{\lambda_{i}}\left(R_{\lambda_{i}}\right)$ $C \bigcup_{k=1}^{m_{0}-1} T_{\lambda_{i}}^{-k}\left(R_{\lambda_{i}}\right)$ and $l\left(\bigcup_{k=1}^{m_{0}-1} T_{\lambda_{i}}^{-k}\left(R_{\lambda_{i}}\right)\right) \rightarrow 0$ as $l\left(Q_{i}\right) \rightarrow 0$ since this set has a fixed number of components independent of $i$.

\section{Proof of Theorem}

A convergent sequence of critical parameter values $\lambda_{n}$ with critical intervals $R_{n, \lambda_{n}}$ will be recursively defined starting with any critical parameter value $\lambda_{0}$ with critical restrictive interval $R_{0, \lambda_{0}}$ (such an interval can be found empirically [11]).

Without loss of generality it may be assumed that the left-hand endpoint of $R_{n, \lambda_{n}}=\left[r_{n, \lambda_{n}}, r_{n, \lambda_{n}}^{\prime}\right]$ is periodic and $\lambda_{n}$ is the right-hand endpoint of a window 
$\left[\lambda_{a, n}, \lambda_{b, n}\right]$. Choose $\varepsilon_{n}$ sufficiently small that (1) $\left(\lambda_{n}, \lambda_{n}+\varepsilon\right)$ contains no window with corresponding period less than $n$ (windows of order $n$ are finite in number, see Guckenheimer [4], Jonker and Rand [7, 8], Milnor and Thurston [10]), (2) for $\lambda \in\left(\lambda_{n}, \lambda_{n}+\varepsilon_{n}\right), l\left(\operatorname{Iorb}\left(R_{n, \lambda}\right)\right)<l\left(\operatorname{Iorb}\left(R_{n, \lambda_{n}}\right)\right)+\frac{1}{n}$, and (3) using Lemma 1 with $\sigma=\frac{1}{n}$ any invariant measure for $T_{\lambda}$ with $\lambda \in\left(\lambda_{n}, \lambda_{n}+\varepsilon_{n}\right)$ has at least half of its mass in a set of lebesgue measure less than $l\left(\operatorname{Iorb}\left(R_{n, \lambda}\right)\right)+\frac{1}{n}$. Then by Lemma 2 , there exists a critical parameter value $\lambda_{n+1} \in\left(\lambda_{n}, \lambda_{n}+\varepsilon_{n}\right)$ such that $l\left(\operatorname{Iorb}\left(R_{n+1, \lambda_{n+1}}\right)\right)<\frac{1}{n+1}$.

Choosing $\varepsilon_{n}$ tending to 0 produces a sequence of intervals $\left(\lambda_{n}, \lambda_{n}+\varepsilon_{n}\right)$ that nest down to some point $\lambda_{\infty}$. Then $\lambda_{\infty}$ will be in the exterior of all windows since $\left(\lambda_{n}, \lambda_{n}+\varepsilon_{n}\right)$ contains no window of corresponding period less than $n$. Furthermore, since $\lambda_{\infty} \in\left(\lambda_{n}, \lambda_{n}+\varepsilon_{n}\right)$, any invariant measure for $T_{\lambda_{\infty}}$ will have a majority of its mass in a set of lebesgue measure less than $l\left(\operatorname{I} \operatorname{orb}\left(R_{n, \lambda}\right)\right)+\frac{1}{n}<l\left(\operatorname{Iorb}\left(R_{n}, \lambda_{n}\right)\right)+\frac{2}{n}<\frac{3}{n}$. Consideration of ergodic components implies that any invariant measure is singular.

\section{References}

1. Arnold, V.I.: Small denominators. I. On maps of the circle onto itself. Izv. Acad. Sci. USSR 138 (2), 255-257 (1961)

2. Collet, P., Eckmann, J.-P.: Iterated maps on the interval as dynamical systems. Boston: Birkhäuser 1981

3. Cornfeld, I.P., Fomin, S.V., Sinai, Ya.G.: Ergodic theory. Berlin, Heidelberg, New York: Springer 1982

4. Guckenheimer, J.: On bifurcation of maps of the interval. Invent. Math. 39, 165-178 (1977)

5. Guckenheimer, J.: Sensitive dependence on initial conditions for one-dimensional maps. Commun. Math. Phys. 70, 133-160 (1979)

6. Jakobson, M.: Absolutely continuous invariant measures for one-parameter families of onedimensional families maps. Commun. Math. Phys. 81, 39-88 (1981)

7. Jonker, L.: Periodic orbits and kneading invariants. Proc. Lond. Math. Soc. (3)-39, 428-450 (1979)

8. Jonker, L., Rand, D.: Periodic orbits and entropy of certain maps of the unit interval. J. Lond. Math. Soc. (2)-22, 175-181 (1980)

9. May, R.M.: Simple mathematical models with very complicated dynamics. Nature 261, 459-467 (1976)

10. Milnor, J., Thurston, W.: On iterated maps of the interval. Preprint (1977)

11. Ruelle, D.: Applications conservant une mesure absolutement continue par rapport à $d x$ sur $[0,1]$. Commun. Math. Phys. 55, 47-52 (1977)

Communicated by J. Mather

Received December 20, 1985; in revised form November 24, 1986 